\title{
Parler aux morts, parler aux ancêtres (Minyanka,
} Mali)

Talk to the Dead, Talk to the Ancestors (Minyanka, Mali)

\section{Philippe Jespers}

\section{OpenEdition}

1 Journals

Édition électronique

URL : https://journals.openedition.org/span/1282

DOI : $10.4000 /$ span. 1282

ISSN : 2268-1558

Éditeur

École pratique des hautes études. Sciences humaines

\section{Édition imprimée}

Date de publication : 1 décembre 1991

Pagination : 181-206

ISSN : 0294-7080

\section{Référence électronique}

Philippe Jespers, «Parler aux morts, parler aux ancêtres (Minyanka, Mali) », Systèmes de pensée en Afrique noire [En ligne], 11 | 1991, mis en ligne le 15 novembre 2013, consulté le 28 juin 2022. URL: http://journals.openedition.org/span/1282 ; DOI : https://doi.org/10.4000/span.1282 


\title{
PARLER AUX MORTS, PARLER AUX ANCETRES
}

\author{
par \\ Philippe Jespers
}

Pour éclairer le rapport qu'en pays minyanka, les hommes établissent entre leurs "morts" et leurs "ancêtres", on pourrait proposer une mise en parallèle de deux types de sacrifice que nous avons eu l'occasion, maintes fois, d'observer, l'un adressé aux morts (dans le cadre des rites funéraires), l'autre aux ancêtres (dans le cadre du culte rendu aux ancêtres lignagers). Le fait que les Minyanka emploient le même terme sungo ("prier") pour les désigner montre qu'à leurs yeux, les deux sacrifices forment un système. Dans cet article, nous allons essayer de montrer qu'on ne peut rendre compte des catégories de "mort" et d'"ancêtre" que par rapport à deux manières de parler aux morts et aux ancêtres selon un rite désigné sous un même vocable, mais correspondant à des "actes de paroles" aux propriétés énonciatives différentes.

Cetie distinction en fait apparaître une seconde qui concerne les actes sacrificiels suivant généralement la prière. Nous montrerons que le rite sacrificiel est lui-même perçu dans l'ordre d'une "voix" ou d'une "parole" attribuable au destinataire (mort ou ancêtre). Une distinction radicale s'imposera: le sacrifice au mort est "sonore", le sacrifice aux ancêtres est "aphone". Que signifie cette opposition? Que nous enseigne-t-elle sur la nature d'une transformation des morts en ancêtres? Telle est la question soulevée dans cette contribution. Mais avant d'y répondre, une présentation des ancêtres s'impose. 


\section{Les sefele (les "ancêtres")}

Pour désigner ce que dans la littérature ethnologique nous appelons les ancêtres, les Minyanka disposent de deux termes: 1) kule: "vieux mort" (de ku "mort" et le "vieux") et 2) sef cle: "vieux maître de l'engendrement" (de se "engendrer", $f \varepsilon$ "propriétaire" et le "vieux").

Cette double dénomination renvoie à l'opposition brousse/village. Les ancêtres sont en effet distribués en deux lieux bien distincts du territoire villageois:

1) en brousse, où ils sont perçus comme kule "vieux morts" dans un "village" dit des "vieux morts";

2) au village où ils sont appréhendés comme sefelع "vieux maîtres de l'engendrement"; mais il faut pour cela que des rites funéraires les aient définitivement installés dans le "village des ancêtres".

De quoi s'agit-il dans ces rites funéraires? De transformer certains parents après leur mort - pas tous les parents, mais seulement ceux qui ont laissé derrière eux une descendance (enfants et petitsenfants), et à condition encore qu'ils ne soient pas morts en situation d'impureté' - en ancêtres de la lignée. La question de l'ancestralité s'articule ainsi à celle des naissances, comme semble bien le signifier le nom de sefelc "vieux maîtres de l'engendrement".

Dans la perspective d'une transformation des morts en ancêtres, on peut considérer que les deux termes kule et sef $\varepsilon l \varepsilon$ permettent, selon le contexte, de désigner les ancêtres en fonction d'un double statut d'existence: ils sont à la fois "ici", dans l'espace du village, comme sefєle, et "là-bas", dans leur propre village de brousse, comme kule. D'où résulte la complexité du culte. Comme ils résident en permanence dans leur village, il faut à chaque fois des rites particuliers (prières, sacrifices...) pour les faire venir en certains lieux du village des vivants; mais ces cérémonies terminées, ils sont censés retourner dans leur village de "vieux morts".

\footnotetext{
'Parmi les vieux parents non ancestralisables, on compte les foudroyés (kle gbo "morts de dieu"), les suicidés ( $\underline{\text { finergbo }}$ ), les victimes de mort subite en brousse, les noyés, etc. Certains ont droit parfois à des funérailles, mais, dans ce cas, il est de toutes façons exclu que l'on fasse passer leur dépouille par la "porte des ancêtres"; on la fait sortir par un trou pratiqué dans le mur d'enceinte, leur interdisant ainsi l'accès au monde des ancêtres.
} 


\section{Les espaces attribués aux ancêtres}

\section{Les cimetières}

Selon une croyance générale, les ancêtres habitent le "village des vieux morts" ( $k u$ kule) situé en brousse, du côté du couchant. Il n'y a pas ici l'idée d'un long voyage à effectuer pour rejoindre un "pays des morts" lointain: pas de rivière à franchir, pas de montagne à gravir, comme le conçoivent d'autres cultures. C'est le cimetière luimême qui constitue le "village des morts". Les défunts y mènent une existence qui est l'exacte réplique de celle qu'ils ont connue dans le village des vivants, comme semble l'indiquer le jeu maîtrisé de la construction des tombes affectant la forme d'une "case" avec une "cour" nettoyée et un champ de mil.

Si l'on se reporte à la topographie d'un territoire villageois, on constate qu'il existe deux sortes de cimetière destinées à deux catégories de défunt:

- le fanga pire, le "petit cimetière", regroupe tous ceux qui sont décédés dans cette tranche d'âge qui va du sortir de l'enfance à la première paternité ou maternité. Ce cimetière est situé à un kilomètre ou deux à l'ouest des murs du village;

- le fangagbo, le "grand cimetière", regroupe les "vieux morts", c'està-dire l'ensemble des parents qui ont atteint durant leur existence cette position d'autorité que leur donne le statut de "vieux père" (tole) ou "vieille mère" (nule). Ce "grand cimetière" est toujours plus éloigné du village que le "petit cimetière", et il se reconnaît généralement à une végétation de brousse plus dense et plus variée, ce qui lui donne souvent l'allure d'un petit bois, appelé le "bois du village des vieux morts".

Soulignons que, dans certains villages, les "chefs de terre" sont enterrés solitairement dans une brousse encore plus lointaine que celle du fangagbo.

Cette hiérarchie des cimetières permet de penser que seuls les morts enterrés au "grand cimetière" deviennent ancêtres. Mais le fangagbo n'est pas pour autant lieu de culte aux ancêtres, sauf en certaines circonstances, principalement lorsqu'il y a eu transgression d'interdits majeurs. On dit alors que les ancêtres ne daignent plus répondre à 
l'appel des "prières" qu'on leur adresse au village. Le responsable du culte des ancêtres met en oeuvre des procédures rituelles d'exception: il fera appel aux sociétés initiatiques du village (Nya, Komo, Nankon...) lesquelles enverront leurs médiums (possédés du Nya ou porteurs des masques du Komo) au village des "vieux morts". Une confrontation aura lieu entre les possédés et les "maîtres du village des morts"; quelques heures plus tard, les possédés reviennent et dictent aux villageois les volontés des ancêtres. Parfois c'est une simple défaillance dans le culte des ancêtres (l'oubli d'une formule, d'une prière...) qui peut constituer le point critique d'une rupture entre les deux villages et exiger, dans de brefs délais, le recours aux médiums des sociétés initiatiques pour régler le conflit.

\section{Le kunyugbo}

Arrivé à proximité d'un quartier de village, l'on ne manquera pas de relever la présence de grandes portes monumentales qui servent de repères pour s'orienter vers telle ou telle maison du quartier. Comme en bien des villages mandé ou voltaïques, chaque maison lignagère est précédée d'un vestibule dont la porte extérieure dresse son fronton vers le ciel.

Le nom commun de ces vestibules est kunyugbo (de kun "mur", nyu "bouche", gbo"grand"), ce qui peut se traduire par "grande porte (bouche) des murs" de la maison. Dans bien des contextes rituels le vestibule se dit aussi "grande porte des ancêtres".

Une série d'indications relatives à l'histoire d'une maison permet de préciser la configuration singulière qui fait la réputation d'un kunyugbo. C'est d'abord l'évocation de la figure de l'ancêtre luimême: toute "porte" est censée perpétuer en son "être" certaines propriétés (physiques et morales) de l'ancêtre comme l'indiquent les noms qui leur sont donnés ("porte du gaucher", "porte du chauve", "porte du rouge"). C'est aussi le rappel de certains interdits édictés par l'ancêtre après sa mort (par exemple, l'interdiction de passage pour les femmes menstruées, pour les familiers convertis à l'Islam . . .) . Enfin, il ressort des règles de la fondation que le kunyugbo se situe dans l'axe exact de l'arrivée de l'ancêtre toujours venu d'ailleurs (d'un autre village, d'un autre pays). Un nom, des interdits, une orientation 
de l'espace..., le kunyugbo apparaît bien comme un "mémorial" de l'ancêtre.

Mais un kunyugbo fait aussi forte impression sur le visiteur par la belle ordonnance de ses lignes architecturales. C'est une oeuvre d'art capable de soutenir la comparaison avec l'architecture que l'on découvre dans la partie nord (islamisée) du Mali. Il serait à peine forcé de dire qu'il marque par l'agencement de ses formes, la décoration de son fronton (pignons phalloïdes, tourelle ogivale...), la signature d'une manière de bâtir propre à tel ou tel clan (Koulibaly, Dembellé, Koné... ). Une classification des kunyugbo, en relation avec l'histoire des clans qui ont essaimé en ce "pays minyanka" reste à faire.

Ainsi chaque maison ( $g b u n$ ) est-elle précédée d'un grand vestibule dont la fonction première est d'assurer la communication entre la cour extérieure et la cour intérieure, autour de laquelle est bâti l'ensemble habité (cases, cuisines, greniers, douchières), entouré d'un mur de clôture qui marque la limite du gbun. Une fois le seuil de la "grande porte" franchi, on découvre une vaste pièce rectangulaire soutenant de ses dix ou douze piliers un toit plat de forme également rectangulaire. La pièce est pour ainsi dire "vide" et il ne viendrait à l'idée de personne de s'y attarder (pour parler, discuter...). Le visiteur sait qu'il trouvera dans la grande cour intérieure un autre vestibule (salle d'accueil, salle à palabres) où il sera attendu par le "maître de maison" (gbunfolo). Si l'on suit le cycle de vie d'un individu (quel que soit son sexe), on peut observer qu'à chaque moment de passage (naissance, circoncision, mariage, décès...), des rites auront lieu au kunyugbo qui traiteront le lien qu'il entretient avec ses ancêtres.

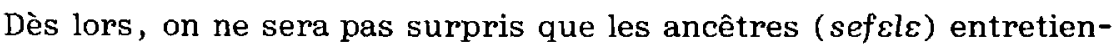
nent avec le vestibule une relation privilégiée. Il y aurait bien, en effet, une relation d'homologie à établir ici entre la fonction du vestibule comme "sas" et la fonction qu'on assigne aux ancêtres comme "passeurs"?.

Mais à cette fonction de passage s'en ajoute une autre. La règle veut qu'à chaque fois que le gbunfolo doit parler aux ancêtres, il se rende au seuil de la porte monumentale du kunyugbo. Nous verrons

\footnotetext{
? Nous empruntons l'expression à Michel Cartry qui l'a appliquée à la fonction du vestibule des ancêtres de la maison gourmantché (Cartry, 1987: 153).
} 
bientôt quelles sont les procédures rituelles qui établissent un rapport entre la parole que le gbunfolo adresse à ses ancêtres en ce lieu et son écoute d'une "voix" issue du monde des ancêtres. C'est là qu'il fera également les offrandes sacrificielles. Une fois l'an, à l'occasion $\mathrm{du}$ poro kworo, la fête des prémices, la "grande porte" doit être entièrement recrépie. Dans la nuit qui précède la fête, un peu avant le lever du soleil, un homme monte sur le toit du kunyugbo et verse en grande quantité de la crème de petit mil qui s'écoulera en de longues traînées d'une blancheur éclatante sur le fronton et les montants de la porte. Cette offrande de "petit mil" de l'année nouvelle est considérée comme célébrant globalement tous les ancêtres de la lignée.

Le kunyugbo assurerait ainsi au moins deux fonctions principales: en jouant sur les deux sens du mot nyu ("orifice"), on pourrait dire qu'il est un organe de naissance (au sens où il fait "passer"), mais aussi un organe qui émet une "voix", la "voix des ancêtres".

\section{Prier le mort}

Le rite que nous allons décrire maintenant s'inscrit dans l'ensemble de la cérémonie d'enterrement. C'est un rite d'adieu aux morts qui s'accompagne d'un sacrifice de chèvre destiné à briser les attaches "acoustiques" du mort au monde des vivants.

La cérémonie "d'enterrement" commence le soir du décès pour se terminer le lendemain au lever du soleil: le mort est enterré dans le fangagbo, le "grand cimetière". La cérémonie se développe suivant plusieurs grandes étapes, dûment nommées, que nous nous proposons de parcourir ici rapidement, en invitant le lecteur à se reporter au relevé topographique du déplacement du défunt de la "maison" à la "tombe".

Ces étapes sont les suivantes:

- Annonce du décès: il faut avertir les parents des villages avoisinants (gbo tyala "mort anoncée"), puis divulguer la nouvelle au village ( $g$ bo jo "dire le mort") par des cris de douleur et des coups de fusil; 
Figure 1: Principales séquence du cérémonial d'enterrement "le". Projection isométrique du quartier Dasiguéla (village de M'pelongosso). Relevé sur le terrain et dessiné par Piet Nankman en 1973.

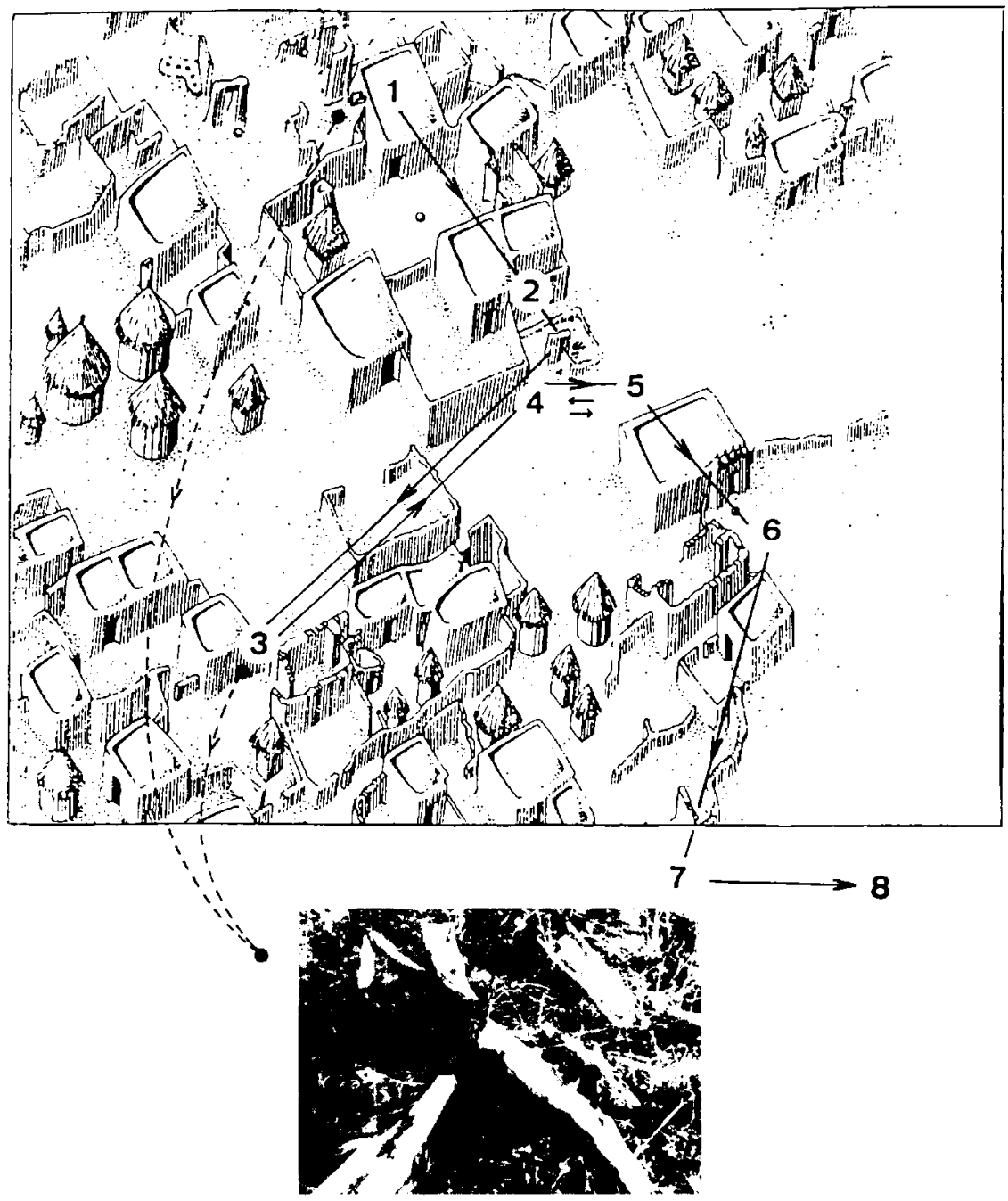

1. gbaleghe: 1ère toilette du mort vieille case

2. n'tyer\&gbo: réclusion du mort grande cuisine

3. kalohgbo: 2ème toilette du mort grande douchière

4. n'tyeregbo:1ère exposition du mort grande cuisine
5. tangbo: 2ème exposition du mort grande cour

6. kunyugbo: "attacher le mort" grande porte

7. kasyoteke: "prier le mort" "place qui arrache la chose"

8. fangabgo: enterrement grand cimetière 
- laver le mort ( $g$ bo wolo yoh pegeni "sortir le mort de sa mauvaise eau") : le défunt est lavé par ses proches dans la "vieille case" (station 1);

- coucher le mort dans la "première cuisine": le défunt y subit une réclusion solitaire avec les ancêtres de la maison (station 2);

- laver le mort (gbowolo): le défunt est lavé et habillé par ses proches dans la "grande douchière" (station 3);

- veillée nocturne par la parenté paternelle et maternelle dans la "grande cour" (station 5);

- danse funéraire devant le mort "exposé";

- franchissement du kunyugbo, "la grande porte des ancêtres" (station 6);

- attacher le mort à son "trois bois" (civière) dans la cour extérieure devant le kunyugbo;

- "prier le mort" ( $g$ bosungo) au lieu appelé kasyoteke: liquidation des dettes du défunt et sacrifice de "la chèvre de l'ouverture de la tombe" (station 7);

- course sur le chemin du fangagbo (le "grand cimetière");

- enterrement (le): introduction du corps dans la "case" (tête à l'ouest, pieds à l'est), nettoyage de la cour attenante à la case, ensevelissement (station 8);

- condoléances à la famille: toutes les filles mariées (kapile) vont passer la nuit dans la maison du défunt.

De ce schéma cérémoniel de l'enterrement, nous retiendrons tout particulièrement le rite dit gbosungo "prier le mort", effectué au kasyoteke (station 7). C'est un rite qui met clairement en jeu une manière de "parler au mort" que nous pourrons, par la suite, opposer à une manière de "parler aux ancêtres".

Après l'avoir vu sortir par la "grande porte", on pourrait s'attendre à ce que le défunt soit immédiatement emporté vers le "grand cimetière" (station 6). Il n'en est rien. L'engagement du défunt sur le chemin du cimetière exige un dernier arrêt rituel. Ce rite, désigné par l'expression u sansyegbo sungo "nous allons prier le mort", comporte trois séquences bien distinctes: 1) "l'adieu du village au mort"; 2) la déclaration par "l'héritier" du rachat des dettes du défunt; 3 ) la 
prièred'offrande d'une chèvreappelée fangutugu yatogo, "quadrupède de l'ouverture de la tombe".

Le terme sungo "prier", qui renvoie explicitement à la séquence sacrificielle, est aussi employé pour désigner l'ensemble de ce procès de parole qui marquera le départ définitif du mort vers le village des ancêtres.

Chaque quartier de village dispose de son propre kasyoteke situé à quelque distance (environ $500 \mathrm{~m}$ à l'ouest des murs de l'agglomération), en un lieu qui marque le début du chemin du cimetière. Le kasyoteke se présente toujours comme un vulgaire terrain vague qui contraste singulièrement avec la belle ordonnance des champs de culture (de mil, de maïs) qui l'entourent. Le terme kasyot $k$ k se décompose comme suit:

- ka désigne une chose au sens le plus large et le plus abstrait du terme (par opposition à ya qui désigne une chose matérialisée sous la forme d'un objet, d'un autel, d'un fétiche...);

- so est un verbe signifiant "enlever", "arracher", que l'on trouve souvent employé dans un contexte d'activités à caractère économique ou juridique (on dira, par exemple, cette "chose" je l'ai arrachée de haute main);

- teke: désigne le lieu, la place.

Nous traduisons donc kasyoteke par "place qui arrache la chose" du mort.

Bien qu'il ait déjà franchi la porte des "ancêtres" (station 6), le défunt ne pourra quitter le village qu'à partir du moment où l'on aura effacé en sa présence les mauvaises paroles, les querelles non résolues, les dettes impayées qu'il laisse derrière lui. Tant que tout cela n'a pas été réglé, il restera au kasyot $k$ ke à mi-chemin entre le village et le cimetière. Il n'est pas rare qu'en certains cas, les deuilleurs fassent appel aux services des possédés des sociétés initiatiques pour négocier avec le mort ce passage délicat par le kasyoteke, car s'il restait lourd d'une dette envers la communauté des vivants, il ne pourrait rejoindre celle des ancêtres, eux dont c'est précisément la fonction d'être les gardiens de la coutume du village.

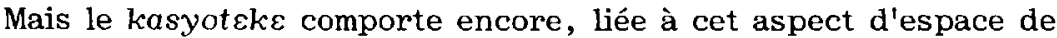
passage, une autre dimension qui va nous ramener plus directement à la question du deuil. Quand on entre dans le kasyoteke, on ne 
manque pas d'être frappé par l'état d'extrême confusion où reste cette place - jamais nettoyée, jamais défrichée - où l'on voit ça et là toutes sortes de détritus : cache-sexe, gouttières, nattes... provenant des rites de toilette et d'habillage des défunts. Si l'on suit attentivement ces derniers rites, on constate que l'usage du kasyoteks comme dépotoir est strictement motivé par le traitement méticuleux dont la gouttière et le cache-sexe du mort ont été l'objet avant son passage au kasyoteke.

Quelle conception se fait-on ici du destin post mortem d'un parent défunt pour qu'on ait besoin de lui faire ses adieux dans cet étrange lieu? Un bref retour vers ces rites va nous permettre de répondre à cette question.

Lors de l'enterrement d'un vieillard, la toilette comporte deux moments importants:

1) le premier lavage, qui se dit u sã syggbowolo yoh pegeni, "nous allons enlever le mort de sa mauvaise eau", a lieu dans le gbahleghe, "vieille case" appartenant à la doyenne du lignage (station 1);

2) le deuxième lavage, qui se dit u sãsye gbo wolo "nous allons laver le mort", a lieu dans le kalohgbo, "grande eau de la chose", la grande douchière de la maison réservée à l'usage des défunts.

Dans la grande douchière, le corps du défunt soutenu par ses frères et par des "parents à plaisanteries" est maintenu en position assise sur la gouttière qui a été arrachée à la "case" où il a été lavé lors du premier rite. Le frère puîné commence par verser de l'eau sur la tête du défunt, imité en cela par les autres deuilleurs. Après quoi, il se prépare à effectuer le rite connu sous le nom de $k a$ loh wolo "verser l'eau de la chose". A l'aide d'une calebasse, il verse un peu d'eau sur les cinq extrémités du corps du défunt dans l'ordre suivant: sur la tête, sur la paume de la main gauche, sur la plante du pied droit, sur la plante du pied gauche, sur la paume de la main droite. Ce geste qui dessine un signe en X (signe de vie, de fécondité) sur le corps marque la fin du rite. L'eau qui a servi à cette opération et à laquelle il est rigoureusement interdit de toucher est évacuée par un trou pratiqué dans le sol au-dessous de la gouttière sur laquelle est toujours assis le défunt. Le fils aîné entre alors en scène. Il s'accroupit devant le corps de son père et, tenant un peu d'eau dans le creux de ses mains jointes, il saisit le sexe du défunt et luj fait subir 
une légère pression tout en esquissant un mouvement d'avant en arrière comme s'il voulait en extraire quelque chose. Il répète ce geste trois fois de suite en veillant à conserver l'eau dans ses mains jointes. Alors, il se lève brusquement et, fuyant droit devant lui (avec interdiction de se retourner), il emporte cette eau jusqu'aux abords de la "grande cuisine" ancestrale. Il se déchausse, entre dans l'enceinte sacrée et verse l'eau en direction des autels de la "grande cuisine".

De retour dans la grande douchière, il incombe encore au fils aîné de fixer le nouveau cache-sexe de son père, cache-sexe qui a été taillé dans un vieux vêtement de couleur rouille d'un chasseur. Et, tandis que les frères commencent à habiller le mort, le fils aîné part avec la gouttière et l'ancien cache-sexe de son père pour aller les jeter au kasyoteke (station 7 ).

Qu'a fait au juste le fils aîné? D'après nos informateurs, l'eau qu'il arrache - par simulacre - du sexe de son père est la dernière eau de sexe du mort. Contrairement aux opérations précédentes effectuées par les autres deuilleurs, il ne s'agit pas seulement d'un rite de purification. Au lieu d'être versée dans la "première douchière", lieu destiné à recueillir la souillure de la mort, cette eau est, en effet, transférée jusqu'à la "première cuisine" qui est, au contraire, un lieu très pur puisqu'un feu y est entretenu pour les ancêtres de la maison. Aussi, seul le fils aîné - il faudrait dire celui qui fut le premier à avoir transformé son père en géniteur (tosegi) - prend ce pouvoir d'extraire du corps de son père, lavé de l'impureté de la mort, cette part de puissance fécondante qui, sous la forme d'une trace d'eau, ne saurait disparaître des murs de la "cuisine des ancêtres".

Toutefois, ce dépôt de la dernière eau de sexe dans la cuisine des ancêtres ne suffit pas à lui seul. Dans l'enchaînement des séquences rituelles, il apparaît comme inséparablement lié à l'obligation pour le fils aîné d'aller jeter l'ancien cache-sexe et l'ancienne gouttière de la case de son père au kasyotcke. On peut penser qu'abandonner en cette parcelle de "terre stérile" les restes de la toilette du père mort, qui symbolisent dans l'association: cache-sexe/gouttière sa puissance fécondante, revient à les stériliser. 
Description d'un gbosungo "prier le mort"

Présentons ici à grands traits le rite que nous avons suivi lors d'un gbosungo effectué lors du décès d'un "maître de maison" que nous avons connu au village de Fungo so (situé à $40 \mathrm{~km}$ au sud-est de Koutiala).

Du kunyugbo (la "grande porte") jusqu'au kasyoteks ("la place qui arrache la chose"), la communauté accompagne la civière en chantant :

"Quitte le village pour la brousse, Sonu (prénom du mort) Quitte le village pour la brousse, Sonu..."

Arrivés au kasyoteke, les porteurs déposent la civière au centre de l'aire en la plaçant selon un axe est-ouest, la tête du mort côté village, ses pieds côté brousse, c'est-à-dire selon un axe qui l'oriente vers le village des ancêtres.

Toute la communauté prend alors place de manière à former un demi-cercle. Au centre, le mort est attaché à son "trois bois", tout enveloppé d'un linceul blanc qui le dissimule au regard des deuilleurs, à l'exception toutefois de sa main gauche: ${ }^{\text {, }}$ qui reste à l'extérieur du linceul, repliée sur sa poitrine. La vue de la main du défunt "chauffe le coeur", dit-on, et pousse certains à manifester leur émotion: on se précipite sur la civière pour saisir "la main du mort", l'embrasser, l'étreindre une dernière fois.

C'est alors au successeur du défunt (son frère puîné) de prendre solennellement la parole. Il dit bien haut que le "temps est venu pour son frère de partir..., que celui qui a une créance le lui dise...", "que, lui, il paiera la dette...", qu'"il ne faut plus tarder, car bientôt ce sera trop tard...". Ces phrases, il les répétera plusieurs fois, avec insistance, mais aucune réponse ne viendra. L'étiquette exige que rien ne soit réglé en présence du défunt, la liquidation effective des dettes se faisant, en principe, quelques jours après l'enterrement, dans le vestibule de la maison du défunt.

L'héritier invite ensuite tous ceux qui se sentent affligés par le "départ de son frère" de venir lui rendre un dernier adieu. Parents,

\footnotetext{
${ }^{3}$ Pour une défunte, l'on sort du linceul la main droite et cela en raison d'un schème corporel propre à la théorie de la personne minyanka qui définit la main gauche corme celle de l'homme, la main droite comme celle de la ferme.
} 
alliés, amis, se succèderont pour échanger une dernière parole avec le mort. Ecoutons, par exemple, le yame "voix de douleur" de la veuve:

"Sonu (prénom du mari)

Je te salue

Mort, tu m'as abandonné

Qui va maintenant nourrir ma bouche?

Ensemble, toi et moi, nous formions une armée

Sonu

Il faut maintenant que tu t'en ailles

J'ai tout organisé pour qu'on puisse te faire

Tes funérailles ("fixer la calebasse")...

Il faut maintenant que tu t'en ailles

Salue bien nos vieux morts et

Que kle (dieu) t'aide à trouver ta place auprès d'eux."

Une chèvre est alors amenée dans le cercle des deuilleurs. On la désigne du nom de fantugu yatogo "quadrupède pour ouvrir la tombe". Dès réception de l'animal, l'héritier s'accroupit devant la civière, puis, posant délicatement la patte gauche de la victime sur la main gauche du défunt, il prononce à voix très haute la "prière" d'adieu:

"Sonu (nom du défunt)

Voici ton fantugu yatogo

Prends-le de jour

Prends-le de nuit

Il ne faut plus que tu reviennes discuter ici avec quiconque A propos de cette chose

Flle t'a été donnée,

Que kle te donne une bonne assise au village de nos vieux morts Sinon toute chose finira."

Cette prière dite, il lâche la patte de la victime et, prenant son coutelas, lui sectionne l'oreille gauche. La chèvre hurle. Sans tarder il jette l'oreille de la chèvre dans la main du défunt, tandis que les porteurs soulèvent déjà la civière et s'engagent à vive allure sur le chemin du "grand cimetière".

Le cri de la chèvre transforme ainsi le kasyoteke en un site sonore qui nous indique qu'il est le lieu d'une étrange violence. La communauté se scinde en effet en deux: l'héritier, après avoir offert la chèvre mutilée au silo, le "premier des fossoyeurs", quitte le kasyot $k$ k, avec les proches parents du défurt, tandis que les autres villageois suivent les porteurs de la civière jusqu'à la tombe. Sur le chemin du "grand 
cimetière", les petites filles essaieront de retourner la civière pour ramener le défunt au village. En vain.

Dans ce rite d'adieu, ce qui doit retenir notre attention, c'est le traitement dont l'oreille de la chèvre est l'objet. Nous nous attacherons à mettre en évidence ce qui s'y élabore d'une transformation d'un mort en ancêtre.

\section{a) L'oreille coupée}

La coupure de l'oreille est liée à la fonction du "cri" de la victime dans la relation que le rite établit avec l'oreille du défunt. Voici en quels termes cette relation est interprétée par les deuilleurs:

"L'oreille du mort, c'est ce qui est le plus difficile à fermer, au moment du décès. Dans la vieille case, on commence par fermer les yeux du mort, ses narines, sa bouche, ses mains. Reste alors l'oreille. On ne peut pas la fermer sauf avec un yame, une voix de douleur! Un parent proche, parfois un ami, entonne un yams. Avec le yame, il dira sa peine, rappelera des situations de vie passée, des qualités que tout le monde reconnaissait au mort... Il faut que l'oreille se ferme ainsi doucement avec des bonnes paroles qui fassent plaisir au mort. Mais quand le cortège arrive au kasyoteke, l'oreille du mort s'ouvre de nouveau. On va la fermer définitivement avec le sacrifice de la chèvre..."

L'affirmation, souvent entendue, que l'oreille est de tous les organes du corps humain celui qui est le plus rebelle à sa fermeture ${ }^{\wedge}$ justifie, aux yeux des deuilleurs, l'obligation de traiter par deux fois l'"oreille du mort" lors du rite d'enterrement. Ce qui, de ce traitement, ressort avec le plus de relief est l'intervention successive de deux "voix" - l'une humaine (celle des chants funéraires), l'autre non humaine (celle du cri de la chèvre) - pour marquer la fermeture définitive de l'oreille du mort. Dès lors, on comprend tou te l'importance du cri de la chèvre: le défunt n'a plus à "entendre" ni à "parler" (donc à être) dans le village, dès le moment où on lui a fait des adieux solennels au kasyot $\varepsilon k \varepsilon$.

\footnotetext{
1 Rappelons, à cet égard, que l'oreille se dit en langue minyanka nqu wi, ce qui peut se traduire littéralement par nqu "bouche" et wi "trou", la "bouche trouée" (sous-entendu: la "bouche" toujours ouverte).
} 
b) L'oreille donnée

Mais ce rite d" adieu" ne s'arrête pas là. Il faut encore rendre compte ici de la fonction visuelle du transfert de l'oreille du corps de la victime vers celui du défunt, et l'image qu'elle suscite dans l'espace du kasyoteke. Le dernier geste du sacrificateur, celui par lequel il "donne" l'oreille, offre au regard des deuilleurs l'image d'un défunt qui, dans le mouvement qui l'arrache à l'espace du village, emporte avec lui une oreille de chèvre vers le monde des ancêtres.

Ces précisions apportées, comment interpréter ce sacrifice de la chèvre? Dira-t-on que le cri de la chèvre est un cri qui tue l'oreille humaine du défunt au moment où on le somme de quitter le village? Peut-on considérer que le geste de placer l'oreille dans la main du défunt soit comme un acte qui anticipe sur sa capacité d'entendre d'une autre manière, avec une "oreille d'ancêtre"?

La façon dont l'héritier traite le corps de la victime nous montre qu'elle est vouée à une sorte de partition brousse/village. La chèvre, une fois amputée de son oreille gauche, est remise au silo, le "premier fossoyeur" qui, à un stade ultérieur du rite, ira l'égorger et la consommer dans son propre quartier". Ce qu'il convient alors de mettre en évidence, c'est l'interdit très strict qui pèse sur le retour de la chèvre vers le village: elle ne peut franchir le seuil d'aucun kunyugbo ("grande porte") au village. C'est dire que le sacrifice de la victime s'accompagne de deux impératifs qu'une analyse ne saurait disjoindre:

1) l'obligation de "donner" l'oreille de la chèvre au défunt en partance vers le "village des ancêtres";

2) l'interdiction de faire passer la chèvre mutilée par un kunyugbo ("grande porte"), à son retour au village.

La conjonction de ces deux impératifs s'explique. Si la chèvre a été choisie pour aider le défunt à franchir l'espace frontière du kasyoteke, on comprend qu'elle n'ait elle-même à franchir aucun kunyugbo, comme si tous les kunyugbo du village étaient devenus, un bref instant, solidaires du non-retour du mort et, par cela même, de son installation dans le village des "ancêtres".

\footnotetext{
s Ici, comme dans bien des sociétés africaines, le rite du creusement de la tombe est caractérisé par une réciprocité générale des services funéraires rendus mutuellenent par deux "moitiés du village" incluant chacune des lignages ou des clans d'origines différentes.
} 
Tout se passe alors comme si cette oreille de chèvre que l'on donne au défunt était l'archétype de la grande porte du kunyugbo. Car c'est bien au seuil de la "grande porte" que le défunt aura à entendre les prières que lui adressera son successeur, quand il aura accédé au statut d'ancêtre. Soulignons, en outre, que les Minyanka considèrent que la chèvre possède une ouïe particulièrement fine.

Mais après la cérémonie d'enterrement", le défunt est encore loin d'avoir acquis ce statut d'ancêtre. Pendant toute cette période, le culte des ancêtres est comme "suspendu"; on dit même que les ancêtres ne répondent plus à la "grande porte" du kunyugbo, et il n'est pas rare qu'une lourde menace pèse sur la maison, une menace qui concerne précisément la non-réouverture de la "porte" du kunyugbo. Pour que les sacrifices aux ancêtres puissent reprendre, il faut que l'ancien gbunfolo apparaisse en rêve pour révéler à son successeur un "message" qui lui permettra de réouvrir la "grande porte" du kunyugbo. De ce message, nous ne savons rien, si ce n'est qu'il s'agit d'une parole forgée dans la langue des ancêtres, et qu'elle se communique par la voie des rêves de gbunfolo en gbunfolo depuis l'ancêtre fondateur. Associée à l'ancêtre fondateur, cette parole est partie constitutive du kunyugbo, et son énonciation à voix très basse dans le seuil d'ombre de la "grande porte" est assurément l'un des moments-clé de la prière; elle permet la réouverture de la communication avec les ancêtres. Autrement dit, le kunyugbo possède ici cette particularité remarquable d'être un espace de rencontre entre le monde des vivants et le monde des morts appelés à devenir ancêtres".

Dès que l'ancien gbunfolo aura ainsi transmis ce "message" à son successeur, il reviendra à ce dernier d'organiser les premières funérailles, le tyere yerenge "fixer la calebasse". Sans entrer dans les détails de cette cérémonie, nous remarquerons qu'elle comporte le sacrifice d'un taureau offert au mort. Le sacrifice se pratique en deux temps: le taureau est immolé par son successeur au seuil de la "grande porte" du kunyugbo, puis il est rôti et bouilli dans le

\footnotetext{
6. un notera que ce rôle de médiateur attribué au dernier "maître de maison" se retrouve également, vien que formulé de manière différente, dans les rites funéraires des Bobo. "Les ancētres, pense-t-on, ne sont pas constament disponibles, ils vaquent à leurs occupations dans le village des morts et c'est à l'un d'eux, à celui qui est le plus récenment décédé, qu'il appartient de rester en contact avec les parents vivants" (Le Moal, 1980: 156).
} 
tyèrègbo, la "grande cuisine" des ancêtres. Le corps de la victime est ensuite coupé en deux, le successeur gardera pour lui la partie gauche de la victime et partagera la partie droite entre les différents lignages du village.

Ce sacrifice du "taureau du mort" assure, dit-on, une double succession:

1) une succession dans le "village des vieux morts": le nouveau disparu rencontre les ancêtres qui lui donnent une place dans leur village;

2) une succession dans le village des vivants: un nouveau sacrificateur prend la place de l'ancien, au titre de "celui qui parle aux ancêtres".

La continuité du culte est ainsi assurée.

Néanmoins, si le nouveau "maître de maison" exerce ses fonctions dès le lendemain des premières funérailles de son prédécesseur, il devra attendre encore plusieurs mois avant de pouvoir le "prier" (sungo) comme ancêtre ( $\operatorname{sef} \varepsilon l \varepsilon$ ). On pense qu'il n'est pas encore bien "installé" dans le village des ancêtres et qu'il faut encore laisser s'écouler un certain intervalle de temps pour que son image s'efface complètement de l'esprit des survivants. On pense même qu'il y a lieu de craindre son retour - tant redouté des femmes et des enfants - dans l'espace du village. Aussi, dès le lendemain des premières funérailles, la fille aînée doit-èlle prendre en charge un rite qui consiste précisément à chasser son père mort. Affublée de la façon la plus grotesque avec son arc et son carquois, elle quitte la maison paternelle en allant "chercher, dit-elle, le mort". Bientôt suivie d'une cohorte de femmes, elle ira de maison en maison, posant inlassablement la même question: "n'avez-vous pas vu le mort?"; elle ne recueillera, en guise de réponse, qu'un "non" et un peu d'argent, dans l'hilarité générale qu'elle provoque en faisant apparaître ainsi l'image de son père.

Une fois ce rite achevé, toute la parenté masculine du défunt s'engage dans une série de rites qui viseront à défaire les derniers liens qui rattachent encore le défunt à la mémoire des vivants.

Ce temps du "travail du deuil" - parfois étendu sur plusieurs mois, voire quelques années - est scandé par deux rites majeurs:

- le sunbwege la "mauvaise médecine de l'arc" (rite au cours duquel le fils casse l'arc, le carquois et le sifflet de chasse de son père). 
- le sikoryatogo "l'animal pour chasser en brousse" (rite au cours duquel tous les fils offrent à leur père un "chien pour chasser en brousse" $)^{?}$.

\section{Parler aux ancêtres}

Au terme du sikoryatogo, on peut considérer que l'image du mort est déconstruite. Dès lors, devient possible le retour de l'ancêtre dans la maison de ses descendants.

Il nous suffira de suivre les étapes du processus qu'enclenchent les prières et les sacrifices effectués dans les lieux de la maison assignés à cette forme de "retour" des ancêtres pour bien en comprendre les modalités. Un de ces rites retiendra notre attention. Avant de l'examiner en détail, il est indispensable de faire une rapide présentation du responsable du culte des ancêtres: le gbunfolo, le "maître de maison".

Le gbunfolo est le membre le plus âgé de la plus ancienne génération $\mathrm{du}$ segment de lignage que constitue le groupe domestique. Témoin vivant des ancêtres, c'est lui qui garde en leur nom les coutumes de la maison et veille au respect des interdits. Cette charge ne lui donne aucun titre particulier; on le nomme parfois "celui qui parle aux ancêtres". Il n'est pas seul à l'assumer, il est obligatoirement șecondé par la doyenne de la maison, la nule, "la vieille mère" classificatoire du lignage. Dans chaque grande maison, sa fonction consisteà préparer les matières oblatoires - eau, crème de mil, bière - que le gbunfolo utilise pour les prières et les sacrifices aux ancêtres.

Mais un troisième personnage interviendra sur la scène rituelle. Le gbunfolo fera, en effet, toujours appel à un "parent cathartique" auquel il cédera sa place au moment d'effectuer les prières et les

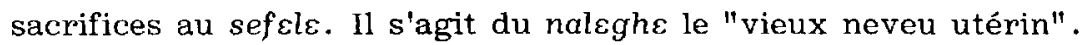

Chez les Minyanka, la catégorie de neveu utérin comprend un ensemble de "parents liés par les femmes": les fils de soeurs, les fils

\footnotetext{
"Nous avons décrit et analysé le premier rite dans "L'arc et le sang du chien" (1983) et le second dans notre thèse (Essai sur la reliqion minyanka), en cours d'achèvement (Université Libre de Bruxelles).
} 
de filles, mais aussi les fils de tantes et de grand-tantes paternelles. Ils sont tous qualifiés de nalo (de na "je" et lo "prendre"). Ainsi, chaque maison a son naleghe attitré, qui est élu selon des critères de sélection très stricts:

- celui de l'âge: il appartient généralement à la génération des nalo issus d'une tante ou grand-tante paternelle (ce qui explique qu'il a souvent l'âge du gbunfolo);

- celui de la respectabilité: on le choisit en tenant compte de ses qualités morales et sociales.

Description d'un sacrifice (ka) au sefele

Nous allons décrire un sacrifice de type courant, en prenant l'exemple d'un rite dit nyere $k a$ "sacrifice de réparation". Le sacrifice doit être effectué un jour faste pour les ancêtres, jour dit sefele sanga "soleil des ancêtres", marquant la fin de la semaine traditionnelle". Quant au nalıghe, le "vieux neveu utérin", il est arrivé dans la maison de ses ancêtres maternels, la veille, de façon à pouvoir être prêt au petit matin du sacrifice prévu.

Le sacrifice a lieu à la "grande porte" du kunyugbo. Les deux hommes ne l'abordent pas de face mais du côté gauche - le premier s'assoit, le dos appuyé au montant de la porte, le deuxième (le sacrificateur) s'accroupit, tourné vers elle, comme si l'un et l'autre s'adressaient à un personnage.

Le nalkghe commence par prononcer d'une voix extrêmement basse la formule secrète (legs de l'ancêtre fondateur) par laquelle il établit la communication avec le monde des ancêtres. Il se prépare ensuite à dire l'invocation aux ancêtres. Celle-ci présente trois propriétés qu'il nous paraît important de dégager si nous voulons saisir la structure énonciative en jeu en cette bouche d'ombre.

Première propriété: le nalıghs énonce, le visage tourné vers le seuil de la "grande porte", les noms de quatre ou cinq ancêtres en commençant par celui du fondateur et en finissant par celui du dernier installé dans le monde des ancêtres. C'est celui-ci, rappelons-le,

\footnotetext{
"La semaine minyanka se compte par six jours, dont chacun correspond, pour un ensemble donné de régions, à un marché qui se tient dans une de ces régions.
} 
qui a transmis la formule secrète de la réouverture de la "grande porte". Deuxième propriété : cette énumération est articulée à voix très basse de façon à ce qu'elle soit rendue inaudible pour l'assistance. Enfin, chaque nom est suivi d'une devise qui interpelle l'ancêtre sur le mode d'une présence-absence. Le nalıghe, au début de la prière, dira à voix très basse:

"Songu (nom du premier ancêtre)

Si tu es là, ton nom est là

Si tu n'es pas là, ton nom est là

Oyoalé (nom du deuxième ancêtre)

Si tu es là, ton nom est là

Si tu n'es pas là, ton nom est là

Nya jigi (nom du troisième ancêtre)

Si tu es là, ton nom est là

Si tu n'es pas là, ton nom est là"

Après cette première invocation aux ancêtres, le nalıghs se présente lui-même, il réitère son invocation, mais cette fois, à voix un peu plus haute et en s'adressant aux ancêtres sous leur nom générique de sefele:

Albyosa ("à présent")

Sefele ("vieux maîtres de l'engendrement")

C'est la vérité

Ce n'est pas en mon nom

C'est en votre nom

Je ne connais pas ma droite

Je ne connais pas ma gauche

Si je suis votre nalo

Vous pouvez arranger cela

Si je ne suis pas votre nalo

Vous ne pouvez pas arranger cela

Voici votre eau

Toute chose, c'est l'eau qui l'arrange...

Alors, joignant le geste à la parole, il verse un peu d'eau à l'aide d'une petite calebasse, une première fois de la main droite (pour les ancêtres féminins), une deuxième fois de la main gauche (pour les ancêtres masculins). 


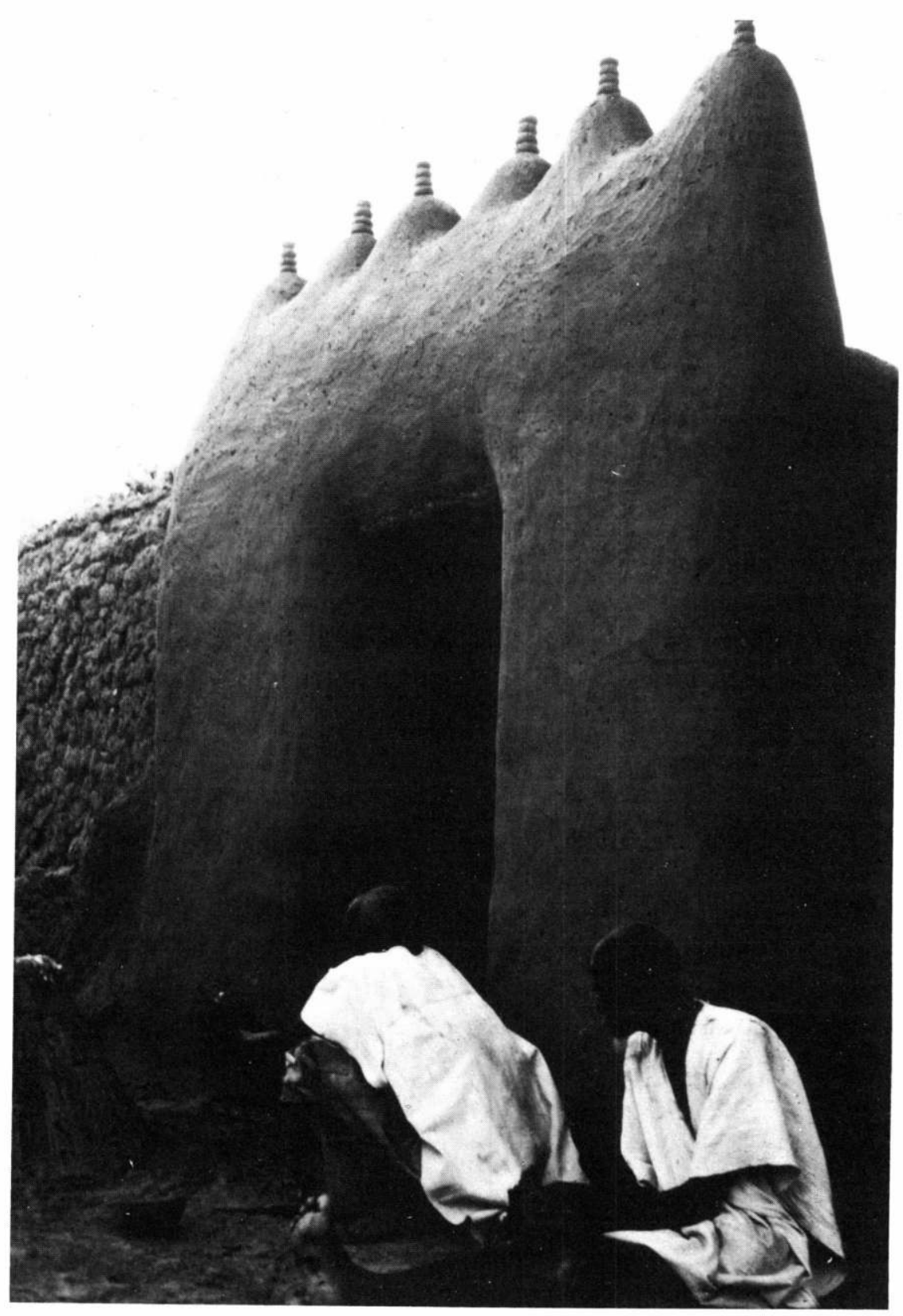

Sacrifice devant la "grande porte" du kunyugbo (Cliché Ph. Jespers) 
L'intonation de la voix joue un rôle essentiel dans l'établissement du dialogue entre le naløgh $\varepsilon$ et les ancêtres. Dans l'enchaînement des deux invocations successives, le nalkghe hausse quelque peu le ton de la voix et utilise le terme albyosa ("à présent"). Cette expression signale une sorte d'événement dans le processus énonciatif : le naleghe entre en scène comme sujet.

En nous inspirant d'une conception "polyphonique de l'énonciation", nous pourrions dire que la prière est bien prononcée par le neveu utérin, mais que la mise en scène rituelle de l'énonciation (voix, succession de paroles ...) implique l'idée, d'une part, que la source de la parole est la "bouche" du kunyugbo et, d'autre part, que le sujet de l'énoncé n'est pas le locuteur (le neveu utérin) mais bien l'allocutaire (l'ancêtre $)^{9}$. L'emploi de la négation dans les énoncés du neveu l'atteste également: "Ce n'est pas en mon nom, mais en votre nom...", "Je ne connais pas ma droite ni ma gauche...".

Vient alors le moment du sacrifice.

Parmi les quarante sortes de volaille répertoriées dans les bassescours minyanka, une seule peut être sacrifiée à la grande porte du kunyugbo: le poulet dit yapara dont le nom est emprunté à la langue bambara. Le terme yapara (traduit d'ordinaire par "chose renversée") désigne une volaille qui, sous une couche superficielle de plumes d'une certaine couleur, cache une robe homogène d'une autre couleur'o. On dit de ce poulet qu'il est yapara parce que sa robe, cachée sous des plumes superficielles, oblige à faire le geste de "retourner" ces plumes pour déterminer son identité. Dès lors, on ne sera pas surpris que le poulet yapara entretienne une relation privilégiée avec la "grande porte" du kunyugbo. Il y a bien en effet une analogie entre ce type de poulet et la sorte de présence-absence des ancêtres au seuil du kunyugbo.

Le sacrificateur saisit d'une main la tête du poulet et la bloque en arrière entre les ailes de façon à bien dégager le cou. De l'autre, il

\footnotetext{
"Dans le cadre d'une conception "polyphonique" de l'énonciation, Oswald Ducrot a mis en cause le fait que la linguistique moderne se fonde sur le préalable de l"unicité du sujet parlant". L'énoncé "est le lieu où s'expriment divers 'sujets' dont la pluralité n'est pas réductible à l'unicité du 'sujet parlant'". (0. Ducrot, 1984).

1o Les Minyanka en arrivent à classer ainsi trois sous-classes de yapara: blanc, noir et rouge, en fonction de la couleur du "dessous" de robe.
} 
prend le couteau sacrificiel et, faisant glisser la lame à plat sur la partie découverte du cou, il dit: "sef $\varepsilon l \varepsilon$, voici pour vos bienfaits". Puis faisant descendre la lame d'un trait oblique il dit: "sef cle, voici pour vos méfaits". C'est en prononçant cette formule qu'il enfonce la lame dans la gorge du poulet. Mais, au lieu de jeter la victime devant la "grande porte", il l'étrangle en lui serrant les ailes autour du cou et en lui comprimant le corps de telle sorte que la mort advienne sans cris ni convulsion. Le sacrificateur colle ensuite sur les deux montants de la "grande porte" quelques plumes arrachées aux extrémités des ailes et à la queue de l'animal.

La mise à mort du poulet yapara déclenche les opérations suivantes. Les petits enfants qui ont assisté à la scène de loin accourent au seuil de la "grande porte" et emportent la victime. La règle veut qu'ils aillent la plumer et la soumettre au feu d'une première cuisson dans la grande cour, en face du kunyugbo. Quelque temps plus tard, ils reviennent et remettent la victime entre les mains du sacrificateur.

Le nalsghe, "vieux neveu utérin", fend le corps de la victime, et dépliant les deux ailes, le maintient à plat, au milieu du seuil de la "grande porte", puis il invite le gbunfolo, le "maître de maison", à lire dans les reins la réponse des ancêtres. La lecture repose sur l'analyse de quatre fígures aléatoires possibles:

blane / blanc

noir / blanc

blanc / noir

noir / noir

Si la réponse est positive (blanc / blanc), le nalıghe dira au gbunfolo: nyere ka tah, "la réparation a eu lieu"; si elle est négative (noir / noir), il dira: nyere ka tah me, "la réparation n'a pas eu lieu". Dans le cas d'une réponse négative (ou mitigée), deux solutions se présentent au gbunfolo: 1) il se rend chez un devin de son choix; 2) le naleghe lui propose tout simplement les services rituels de son propre fils qui, dans cette circonstance, est appelé nalo sya saga "le neveu recherché pour toute chose". Le fait qu'on assigne au jeune nalo une tâche rituelle que n'a pu mener à bien le vieux nalo renvoie 
sans doute à la connivence qu'en raison de son statut de voleur rituel le jeune nalo entretient avec les ancêtres de la maison' '.

La mort sans cri de la victime ainsi que la rétention des voix dans l'espace de ce sacrifice (celle du sacrifiant comme celle de la victime) apparaissent comme un code spécifique traitant la "présence" des ancêtres sur fond "d'absence". Mais de cette absence, il ne faut pas conclure à une absence de réponse. Nous l'avons vu, c'est le déchiffrement silencieux de la couleur des reins de la victime qui permet la formulation de l'oracle. Comme l'explicitent les anciens: "c'est le poulet yapara (chose renversée) qui dit la vérité et dit les paroles des sef $\varepsilon l \varepsilon^{\prime \prime}$.

Entre une prière (sungo) adressée à un mort (pour qu'il quitte le village) et une prière (sungo) adressée à un ancêtre (pour qu'il se fasse entendre au village) il existe des correspondances qui permettent de les traiter comme les éléments d'un même système symbolique.

La prière adressée aux morts est dite à voix haute, avec véhémence; le locuteur parle en son nom, par phrases impératives. La prière adressée aux ancêtres est dite à voix basse, avec déférence; le locuteur parle au nom de l'autre, par phrases de soumission.

De même s'opposent les actes sacrificiels qui suivent les deux types de prière. Il ressort que le sacrifice est perçu dans l'ordre d'une "voix" ou d'une "parole" attribuable au destinataire.

Le sacrifice aux morts demande un cri de la victime qui fait advenir l'absence du mort sur fond de présence, celui aux ancêtres s'accomplit sans cri, le silence de la victime faisant advenir la présence de l'ancêtre sur fond d'absence. L'analyse comparative de ces deux rites permet ainsi d'établir que c'est bien une "manière de parler" et une "manière de sacrifier" qui donne réalité aux ancêtres.

Qu'au vieux neveu utérin qui, dans la "bouche d'ombre" du kunyugbo, invoque à voix très basse les ancêtres, ne réponde que le silence, c'est là le trait le plus éloquent de la prière qu'il leur adresse. C'est seulement avec la formule par laquelle il se présente devant les ancêtres que le neveu utérin fait entendre sa propre parole, alors que jusque là il l'avait retenue pendant toute l'invocation. Il

11 Le neveu utérin perd son statut de voleur rituel au fur et à mesure de son avancée en âge. 
faudrait de longs développements pour montrer que les Minyanka ont élaboré une classification des paroles d'où il ressort que le silence n'est pas une absence de parole, mais qu'il est "sous elle" comme une source de paroles. L'ancêtre répond, mais sa réponse est à lire dans des traits prélevés sur le corps de la victime dans un système aléatoire de signes. Il est donc bien permis de penser que, chez les Minyanka, la transformation d'un mort en ancêtre s'accomplit par une transmutation de la voix et de l'écoute.

Philippe Jespers

URA 221

\section{Références bibliographiques}

Cartry, Michel

1987 "Le suaire du chef", in Sous le masque de l'animal. Essais sur le sacrifice en Afrique noire, M. Cartry éd., Paris, PUF, 131-231

Ducrot, Oswald

1984 I e dire et le dit, Paris, Les éditions de Minuit.

Hertz, Robert

1928 "Contribution à une étude sur la représentation collective de la mort" in Mélanges de Sociologie Religieuse et Folklore, Paris, Félix Alcan (1ère éd. 1907)

Jespers, Philippe

1983 "L'arc et le sang des chiens", Systèmes de Pensée en Afrique noire, 6, Paris, EPHE-CNRS, 65-102

Jonckers, Danièle

1987 La société minyanka du Mali, Paris, L'harmattan, ("Connaissance des hommes").

Le Moal, Guy

1980 Les Bobo. Nature et fonction des masques. Paris, ORSTOM. 